\title{
Device telemonitoring may observe emotional status - psychological impact of arrhythmic episodes alert.
}

\author{
Aneta Fronczak ${ }^{1, \mathrm{~B}-\mathrm{E}}$, Damian Łasocha ${ }^{1, \mathrm{~B}, \mathrm{E}}$, Anna Mierzyńska, ${ }^{1, \mathrm{~B}, \mathrm{E}-\mathrm{F}}$, Maciej Sterliński ${ }^{1, \mathrm{~A}-\mathrm{F}}$ \\ A - Research concept and design, B - Collection and/or assembly of data, C - Data analysis and interpretation, \\ D - Writing the article, E - Critical revision of the article, F - Final approval of article
}

1 Institute of Cardiology, Warsaw, Poland

Address for correspondence:

Aneta Fronczak, Institute of Cardiology, Warsaw, Poland

email: afronczak@ikard.pl

Damian Łasocha, Institute of Cardiology, Warsaw, Poland

email: dlasocha@ikard.pl

Anna Mierzyńska, Institute of Cardiology, Warsaw, Poland

email: amierzynska@ikard.pl

Maciej Sterliński, Institute of Cardiology, Warsaw, Poland

email: msterlinski@poczta.onet.pl

Received: 28.12.2016

Revised: 29.12.2016

Accepted: 29.12.2016

\section{Key words:}

Telemedicine and e-health constitute a modern and quickly developing approach to health care provision ${ }^{(1)}$. There is evidence for telemonitoring of cardiac implantable electronic devices (CIED) to improve the prognosis in patients with CIED, and remote multiparameter monitoring based on defibrillation devices has become a class II $b$ recommendation in those subjects ${ }^{(2,3)}$. Telemedicine has also been shown to be helpful to manage psychological status in chronic diseases, and internet-mediated interventions are believed to be effective for depression and anxiety ${ }^{(1,4,5)}$. Mental disorders related to life with an implantable cardioverter-defibrillator (ICD) are a known and common problem ${ }^{(3)}$. We present the case of a tele-transmission report that led to revealing ICD-related stress disorder aggravation. A male patient aged 58 years with heart failure, dilated cardiomyopathy and a history of embolic ST elevation myocardial infarction, severe left ventricular systolic dysfunction and permanent atrial fibrillation (AF) underwent implantation of a single chamber St. Jude Medical ICD Fortify VR 1233-40 for primary sudden cardiac death prevention in 2013. The next year he suffered multiple inadequate cardioverter-defibrillator shocks prompted by AF with a high ventricular rate, which was the reason to provide psychological care due to distress and depressive symptoms regarding recent arrhythmic events. Reprogramming of the device was done, with the active ventricular fibrillation (VF) zone only ( $230 \mathrm{bpm} / 20$ intervals) and the ventricular tachycardia (VT) zone set to monitor mode (151 bpm/30 intervals). In November 2016, due to safety recommendations the patient was equipped with a transmitter and started being supervised by the St. Jude Medical Merlin.net system ${ }^{(6)}$. The patient has never before experienced malignant true ventricular arrhythmias to be treated by ICD.

As text message about a red alert indicating VT episodes occurred, and an immediate transmission analysis showed in total 9 episodes of AF with a rapid ventricular response - three were classified as VT with no therapy and 6 of them were correctly diagnosed as supraventricular tachycardia (SVT). A list of transmitted arrhythmic episodes is shown in Figure 1, the 


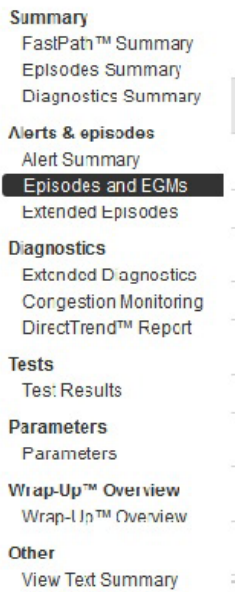

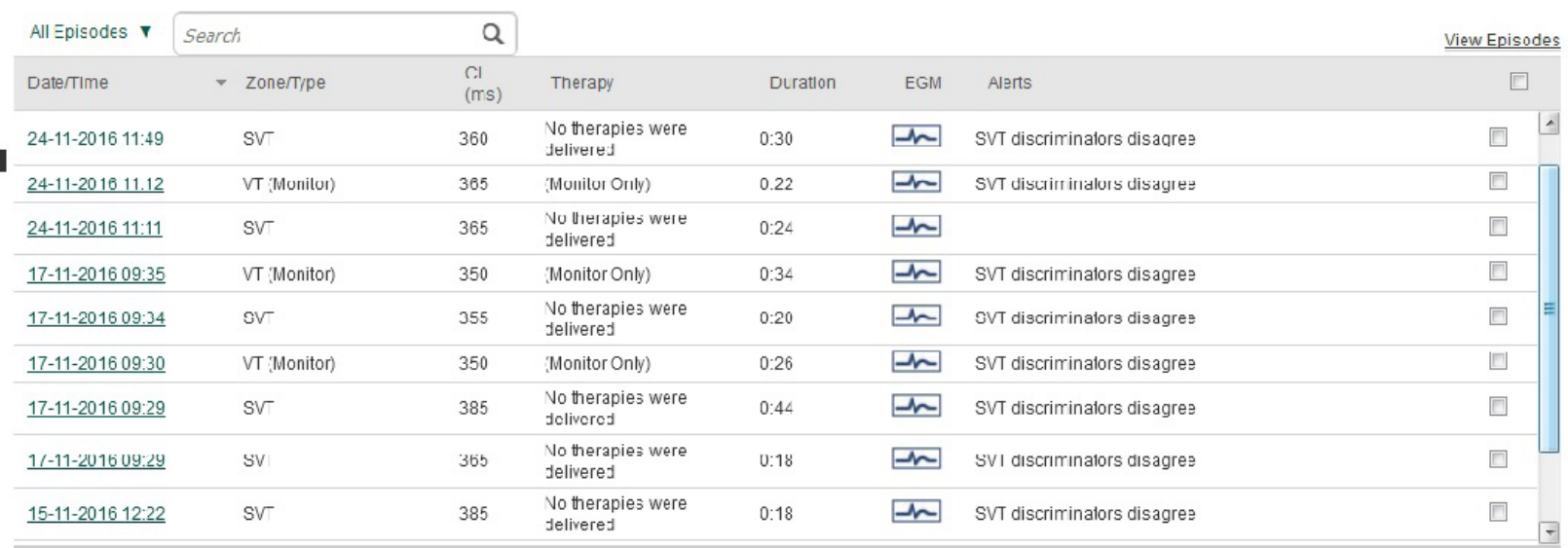

Figure 1 Transmission dated 25 of Nov. 2016 with list of tachyarrhythmic episodes that occurred between 15 and 24 of Nov. 2016.

decision tree of one of the episodes, classified as SVT, is shown in Figure 2, and the intracardiac electrogram of this episode

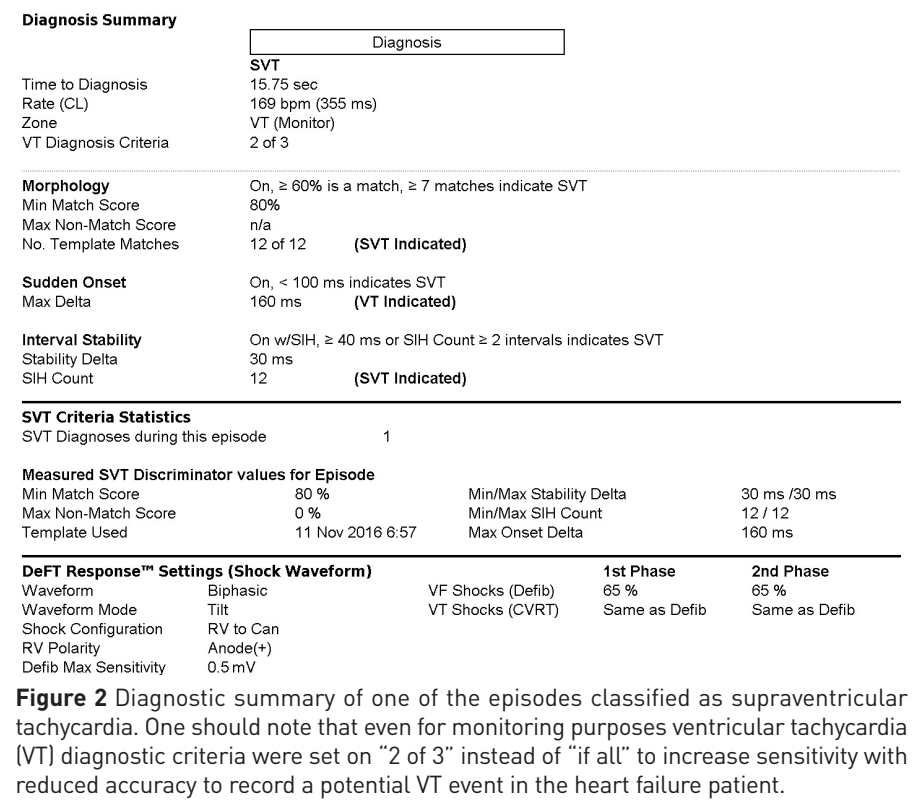

is shown in Figure 3. Because the episodes appeared after a long period of absence and formed two bursts (8/9 episodes within 2 days), phone contact was made to check the patient's actual status. No acute illness was reported but there was an increase in anxiety, which was presumed as a potential adrenergic trigger for events with a probable mixed background: previous anxiety, unscheduled safety action, ICD discharge reminds and closing in-hospital examinations. A prolonged half-hour conversation was confirmed by the patient to be curative and calming for his distress, and following this an additional psychological service during hospitalization one week later was provided. To definitely eliminate rapid conduction as a cause of inadequate discharges, an atrioventricular node ablation with an ICD upgrade to cardiac resynchronization therapy was considered, but this management was discussed and taking into consideration the provided mental care was temporarily postponed.
The postulated main advantage in device telemonitoring is to provide a fast diagnosis and therapeutic actions that may

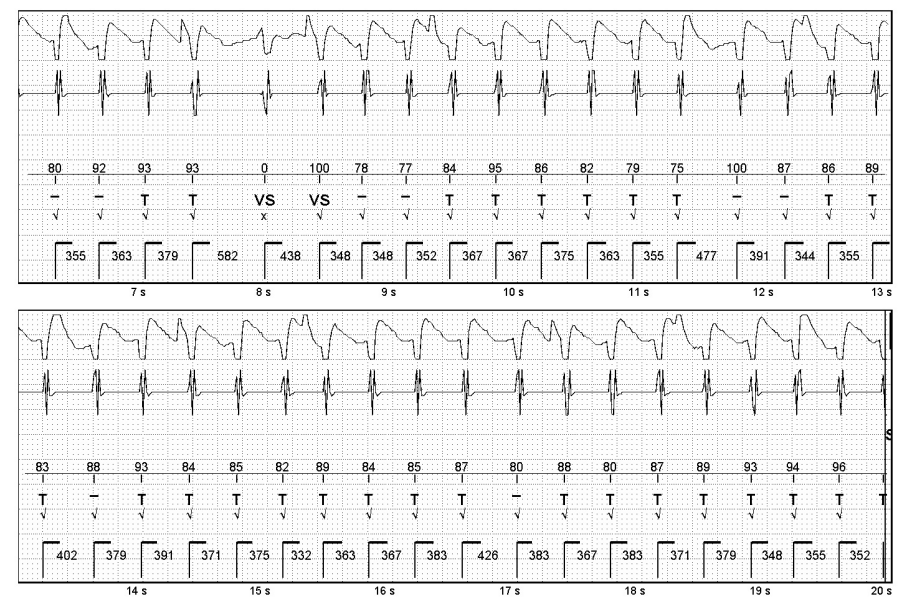

Figure 3 Intracardiac electrogram of an episode of atrial fibrillation with a rapid ventricular response classified by the actual device settings as supraventricular tachycardia.

be crucial to prevent life-threatening events ${ }^{(7)}$. The significance of these actions is commonly well established for the following end-points: VT/VF, atrial fibrillation, pacing percentage changes or mechanical/electrical failure of the leads and device. Thus telemedicine affects in an obvious way patients' quality of life. The underlying diagnosis may be hidden sometimes beneath those factors: an acute mental disorder was represented by arrhythmic events and required immediate care. Psychological intervention provided relief and may be the bridge to delayed upgrade and ablation procedures. We have not found in the literature related reports. Last but not least, it should be emphasized that a high-rate strategy of ICD programming ${ }^{(8)}$, though disputable, turned out in this case to be crucial for the patient to prevent harmful discharges caused by supraventricular arrhythmias.

Conflict of interest: AF, DE, AM - none, MS - consulting and lecture fees from Biotronik, Medtronic and St. Jude Medical, PI in Medtronic and Zoll trials. 


\section{References}

1. Winslow BD, Chadderdon GL, Dechmerowski SJ et al. Development and clinical evaluation of an mHealth application for stress management. Front Psychiatry. 2016 Jul 26;7:130. doi: 10.3389/fpsyt.2016.00130.

2. Hindricks G, Taborsky M, Glikson M et al.; IN-TIME study group. Implant-based multiparameter telemonitoring of patients with heart failure (IN-TIME): a randomised controlled trial. Lancet 2014; Aug 16;384(9943):583-90.

3. Ponikowski P, Voors AA, Anker SD et al. Authors/ Task Force Members; Document Reviewers. 2016 ESC Guidelines for the diagnosis and treatment of acute and chronic heart failure: The Task Force for the diagnosis and treatment of acute and chronic heart failure of the European Society of Cardiology (ESC). Developed with the special contribution of the Heart Failure Association (HFA) of the ESC. Eur J Heart Fail. 2016 Aug;18(8):891-975.

4. Klein JP, Gerlinger G, Knaevelsrud C et al. Internet-based interventions in the treatment of mental disorders: Overview, quality criteria, perspectives. Nervenarzt. 2016 Nov;87(11):1185-1193.

5. Mochari-Greenberger H, Vue L, Luka A, et al. A tele-behavioral health intervention to reduce depression, anxiety, and stress and improve diabetes self-management. Telemed J E Health. 2016 Aug;22(8):624-30

6. http://www.fda.gov/MedicalDevices/Safety/AlertsandNotices/ucm524666.htm

7. Varma N, Pavri BB, Stambler B, et al.; TRUST Investigators. Same-day discovery of implantable cardioverter defibrillator dysfunction in the TRUST remote monitoring trial: influence of contrasting messaging systems. Europace. 2013 May;15(5):697-703. doi: 10.1093/ europace/eus410.

8. Wilkoff BL, Fauchier L, Stiles MK et al. Document Reviewers. 2015 HRS/EHRA/APHRS/SOLAECE expert consensus statement on optimal implantable cardioverter-defibrillator programming and testing. Europace. 2016 Feb;18(2):159-83 